\title{
Hormonotherapy in the Management of Recurrent Ovarian Cancer
}

\author{
Mehmet Kulhan ${ }^{1}$, Nur Gözde Kulhan ${ }^{2}$, Ümit Nayk1 ${ }^{3}$, Cenk Nayk1 ${ }^{4}$,Paşa Uluğ $\breve{g}^{5}$, \\ Nahit $\mathrm{Ata}^{6}$, Hülya Toklucu ${ }^{7}$ \\ ${ }^{12,3,4,5,6,7}$ (Gynaecology and Obstetrics Department/Erzincan University Medical Faculty/ Erzincan, \\ Turkey) \\ ${ }^{1,2}$ (Tepecik Obstetrics and Gynaecology Training and Research Hospital, Obstetrics and Gynaecology Unit,
}

Izmir, Turkey.)

\begin{abstract}
The treatment of patients with recurrent ovarian carcinoma after failure of first and second-line chemotherapy is still debated. Chemical agents used for third and fourth-line therapy usually yield poor results with severe toxic side effects.

Objective: To summarize our experience with Tamoxifen and megestrol in the treatment of patients with recurrent ovarian cancer.

Methods: From September 1995 to June 2015 we administered Tamoxifen and Megestrol to 29 patients with advanced and recurrent epithelial ovarian cancer.

Results: There was no complete responder in patients taking tamoxifen. One (1/15) patient who used tamoxifen achieved a partial response lasting 12 months. There was also no complete responder in patients taking megestrol. Three patients (\%21.4) who used Megestrol achieved a partial response but eleven \% 78.57 (11/14) had no response from the treatment.

Conclusion: Hormonotherapy has approximately $\% 10$ answer rate. There is a correlation between the answer given to hormonotherapy and the existence of hormone receptors. In general, the answer given to hormonotherapy is at a low level. At present, it is used as an alternative treatment option in the patients who do not answer to chemotherapy or who cannot tolerate the toxic effects of chemotherapy.

Key words: metastatic epithelial ovarian cancer, hormone therapy, tamoxifen, megestrol chemotherapy failure
\end{abstract}

\section{Introduction}

Ovarian cancer is the leading cause of death from all gynecologic malignancies. Ovarian cancer is characterized by its insidious onset and lack of early specific symptoms. About two-thirds of patients with ovarian cancer will present in International Federation of Gynecology and Obstetrics (FIGO) stages III and IV, having widespread tumor dissemination in the abdominal cavity, with or without varying degrees of pleural effusion. The prognosis of these patients remains poor, with 5-year actuarial survival of $23 \%$ and $14 \%$ for FIGO stages III and IV, respectively $(1,2)$. Management of these patients consists mainly of primary debulking surgery followed by platinum-based chemotherapy (2).

Over the past several years, combinations of platinum and taxol became standard in the primary management of advanced ovarian cancer (3). Despite a primary response rate of up to $70 \%$, the majority of patients suffer relapse (4). Unfortunately, regimens used as second-line therapy in such situations have a low response rate and are associated with severe side effects that negatively influence the quality of life and necessitate long stays in hospital and intensive palliative care. It is therefore important to explore alternative treatment modalities that have cost-benefit ratios favorable in terms of toxicity. Hormonal therapy perhaps best fulfils these requirements. Epidemiologic studies, animal experiments and receptor studies show that not only normal ovaries but also many malignant ovarian tumors can be considered to be endocrine related and hormone dependent. The aims of this study were to validate the efficacy of hormonotherapy in the management of recurrent ovarian cancer.

\section{Material method}

Our study was conducted between September 1995 and June 2015 in the İzmir Tepecik Research and Training Hospital. 29 women with recurrent ovarian cancer were evaluated retrospectively. These women included patients who: a) had relapsed following at least two lines of chemotherapy, b) were considered too ill to receive or to continue to receive chemotherapy, or c) refused to continue chemotherapy for any reason. All had histologically or cytologically proven epithelial ovarian cancer. All patients had normal blood count, liver and kidney function tests. Disease was evaluated clinically and by ultrasound or Computerized Tomography (CT). No age or performance status limit was imposed. CA-125 measurements were performed before treatment 
and once a month thereafter. Disease status and response were evaluated by clinical examination, sequential CT scanning and CA-125 measurements. Response was classified as complete response and partial response, stable disease and progressive disease. The Standard response criteria were determined according to World Heath Organization criteria.

Statistical analyses have been done by using "Windows operating system compatible SPSS 13.0" software. The results are explained as average standard deviation $\pm(\mathrm{SD})$ for the chronic variables. Student's $t-$ test was used to compare the chronic variables while k-square test was used to compare nonpermanent (categorical) variables. It is accepted as meaningful that the quantity of $\mathrm{P}$ is $<0.05$.

\section{Results}

583 patients were diagnosed with ovary carcinoma and 29 (\%4.97) of them have been applied with hormonotheraphy. The age distribution of the patients with ovary cancer who were included in the study is between 43 and 84 and the average age was decided as $60 \pm 10.09$ (average $\pm \mathrm{SD}$ ). The most frequent complaint was identified as abdominal hoove. Sixteen patients had serous cystadenocarcinoma, eight patients had mucinous carcinoma, and five patients had undifferentiated carcinoma (Table 1). When the patients were examined with regard to patient history, internal diseases and other cancer types, \%13.79 (4/29) of the patients were diagnosed with hypertension and diabetes mellitus. There were no extra diseases in other patients. It was identified that none of the patients had secondary malignity. All of the patients included in the study had stage 4 cancer. Tumor site was abdominal with pelvic spread in 15 patients ( 3 of them with pleural effusions), liver metastases in 5 patients, lung metastases in 4 patients, and liver and lung metastases in 5 patients. All of the patients also had ascites.

All cases have primarily been applied debulking surgery and only one patient had cytoreductive surgery (\% $3.44(1 / 29))$ because of the recurrent mass. 14 patients $(\% 48.27(14 / 29))$ with recurrent ovary cancer were treated with $160 \mathrm{mg}$ Megestrol acetate and 15 patients (\% 51.72 (15/29)) were treated with $20 \mathrm{mg}$ tamoxifen for a year. It is determined that hormonotheraphy has been applied by an average of 3.8 $\pm 2.9(1-12)$ months.

There was no complete responder in patients taking tamoxifen. three (3/15) patient who used tamoxifen achieved a partial response lasting 12 months. These patients had lung metastases and abdominal disease. Four years previously they underwent total abdominal hysterectomy and bilateral salpingo-oophorectomy for stages IIb, IIIc and IIIa carcinoma of ovary. They received six courses of cisplatin and cyclophosphamide. Lung and abdominal metastases were diagnosed 10 month prior to tamoxifen treatment. They received treatment with carboplatin and paclitaxel and achieved minimal response for 8 months. After progression, the patients received cyclophosphamide for 2 months. Treatment was stopped because of deterioration in their general condition and they subsequently responded to treatment with tamoxifen. Response was evaluated by repeated CT scans. In three patient (\%20) the disease stabilized for a median of 12 months. In the other twelve patients $(\% 80)$ the disease progressed and during the treatment, one of the patients deceased in the early period. There was also no complete responder in patients taking megestrol. Three patients (\%21.4) who used Megestrol achieved a partial response but eleven \% 78.57 (11/14) had no response from the treatment. These patients that achieved partial response had advanced ascites and were bedridden for more than $90 \%$ of the time. Chest and abdominal CT showed huge ascites and peritoneal spread. Mammography and gastrointestinal analysis were normal. CA-125 valuses were 500 proximately. An abdominal puncture was performed. Cytologic examination of the ascites revealed serous ovarian cystadenocarcinoma cells. After 12 months of megestrol treatment they achieved a clinically partial remission. CA-125 valueses decreased and their abdominal CT scan showed no progression but minimal regression. In a case with advanced malignity, secondary cytoreduction was performed and then 160 $\mathrm{mg} /$ day megestrol acetate was started. The patient did not respond to the this treatment.

\section{Discussion}

The optimum treatment of patients with recurrent disease remains a major management problem in ovarian cancer. Subsequent treatment is based on the timing and nature of the relapse and extent of prior therapy (5). Most authors agree that patients who respond to initial therapy and relapse more than 6 months later have disease that is most likely still sensitive to prior drugs. Patients with tumor progression during treatment, or who have disease that is likely resistant to the initial drugs, should receive alternative drugs (6). Most drugs used in the treatment of recurrent disease are platinum agents and paclitaxel as single agents or in combination in drugsensitive patients, with a response rate of $30 \pm 40 \%$ (7). In the management of drug-resistant patients and after failure of repeated chemotherapy in drug-sensitive patients, there is no consensus as to what drug to use as second-line therapy. No studies of combination chemotherapy have been conducted, and single-agent therapy is generally chosen. Several new agents have shown substantial activity in the treatment of patients with recurrent ovarian cancer. These drugs include oral etoposide, docetaxel, topotecan, vinorelbine, liposomal doxorubicin, gemcitabine, tamoxifen and megestrol acetate, with response rates of $13 \pm 30 \%(13 \pm 19)$. Patients who were 
previously heavily dosed with chemotherapy and have disease progression or recurrence are incurable by other means and would be expected to have a poor response to second and third-line cytotoxic treatment. In these patients, palliation is the goal. Hormonotherapy provide a novel approach to treatment.

The results of receptor and in vitro studies have also shown that ovarian cancer is an endocrine-related cancer $(8,9,10)$. In a comprehensive analysis of 52 studies, reported the presence of estrogen (ER) and androgen receptors (AR) in the majority of primary ovarian cancers (63\% and 69\% respectively). Progesterone receptors (PgR) were also found in about 50\% of tumors (11). In our study, while all of the patients who were started on hormonotheraphy have positive ER and PR receptor examination, the patients with no ER and PR receptor acceleration were not started on hormonotheraphy.

Many hormonal agents have been used in the treatment of ovarian cancer. Among these agents, Selective estrogen receptor modulators (SERMs), Estradiol (E2), progesterone, Androgen and gonadotropinreleasing hormone (GNRH) analogues can be listed. In advanced ovary cancer, answer rates to the hormonotheraphy done with progestin are between $\% 8$ and $\% 15(12,13,14)$. In clinical studies, the most worked on molecule is tamoxifen. In pre-clinical studies, it is shown that tamoxifen blocks the multiplying of ovarian cells in vitro environment. Tamoxifen competitively inhibits the estrogen binding by binding itself to the estrogen receptors. Tamoxifen - estrogen - receptor complex is bound to DNA but which one of the agonistic, estrogenic or anti-agonistic, anti-estrogenic conduction will be dominant is determined by the promotor component existing in that specific cell type(15).

In many studies, tamoxifen has been used as the only agent in advanced ovary cancer $(16,17,18)$. Paraz Gavcia and Carrasco examined the studies in which tamoxifen was used as the only agent in the patients with advanced ovarian cancer and they confirmed that most of the patients got treatment before and they did not respond to chemotheraphy(19). 648 patients were examined and it was reported that the answer rate was $\% 13$. $\% 3$ full answer and $\% 9$ partial answer rates have been found. The cancer was stable in $\% 38$ of the patients. These results are not exactly different from the results retrieved with progestin(19). All members of the patient group included in our study had chemotherapy and they showed similar characteristics with regard to nonresponsiveness to chemotherapy.

In a Gynecologic Oncology Group (GOG) study, it has been reported that with the usage of $20 \mathrm{mg} / \mathrm{day}$ of tamoxifen, which is twice the dosage used in breast cancer, $\% 13$ full remission rate has been obtained. 8 out of 9 patients with high estrogen receptor rate, full remission has been obtained(20,21). In another extensive study; chemohormontheraphy was examined in the patients who had not received any treatment with advanced disease, 100 patients have been treated with the combination of cisplatin and doxorubicin, and half of these patients have been concurrently started on tamoxifen. However, no difference could be found between two groups with respect to survey and survival without malignity (19). In our study, while partial answer is received form $\% 20(3 / 15)$ of five patients using tamoxifen, no answer received to the treatment from \%80 (12/15) of the patients.

Although the data regarding effects of androgens and progestins are less extensive, Rose \& Barnea reported(22) the antiprogestin mifepristone to downregulate progesterone expression on human ovarian cancer cell lines. Mifepristone blocked cells in a G0/G1 phase and thus reduced the number of cells in the S phase. The efficacy of mifepristone was compared with that of taxol and tamoxifen in the same ovarian cancer cell line. Continuous exposure to tamoxifen resulted in a varied cytostatic response and in a transient change in the cell cycle. Taxol inhibited growth of some but not all cancer cell lines

Using chemotherapic agents, tamoxifen or other hormonal agents as salvage treatment in the patients with advanced ovary tumors is a huge study field for researchers. Hormone receptor levels and prognostic factors over the surface of the tumor are not correlated in the patients of ovary cancer. However, receptor condition is used to anticipate the answer that will be given to the hormonal agents before hand. In order to reveal the hormone receptors which are the predicator of hormonal activity on the surface of ovarian tumor, randomized and controlled studies need to be done(23).

\section{Conclusion}

Hormonotherapy has approximately $\% 10$ answer rate. There is a correlation between the answer given to hormonotherapy and the existence of hormone receptors. In general, the answer given to hormonotherapy is at a low level. At present, it is used as an alternative treatment option in the patients who do not answer to chemotherapy or who cannot tolerate the toxic effects of chemotherapy.

"The authors declare that they have no conflict of interest."

"All procedures performed in studies involving human participants were in accordance with the ethical standards of the institutional and/or national research committee and with the 1964 Helsinki declaration and its later amendments or comparable ethical standards."

"This article does not contain any studies with human participants or animals performed by any of the authors." 


\section{References}

[1]. Pettersson F Annual Report on the Result of Treatment in Gynecological Cancer. Stockholm, Sweden:1994:102

[2]. Makar AP, Baeklandt M, Trope CG et al. The prognostic significance of residual disease, FIGO substage, tumor histology and grade in patients with FIGO stage III ovarian cancer. Gynecol Oncol. 1995;56(2):175-80.

[3]. Bats AS, Barranger E. Management of ovarian cancer. J Gynecol Obstet Biol Reprod (Paris). 2008 Jul;37 Spec No 2:F64-8.

[4]. FG Lawton, JP Neijt \& KD Swenerton Optimum chemotherapy regimens, dose intensity, and new drugs. In Epithelial Cancer of the Ovary. BMJ Publishing Group. London 1995:186-209.

[5]. McGuire WP, Ozols RF. Chemotherapy of advanced ovarian cancer. Semin Oncol 1998;25:707.

[6]. Güth U, Kann SR, Huang DJ, Schötzau A, Holzgreve W, Wight E. Systemic therapy developments and their effects regarding the current concept of recurrent ovarian carcinoma as a chronic disease. Arch Gynecol Obstet. 2009 Nov;280(5):719-24.

[7]. Markman M, Rothman R, Hakes T, et al. Second-line platinum therapy in patients with ovarian cancer previously treated with cisplatin. J Clin Oncol 1991;9:389-93.

[8]. Gadducci A, Genazzani AR. Endocrine therapy for gynecological cancer. Gynecol Endocrinol. 1999 Dec;13(6):441-56.

[9]. Galtier-Dereure F, Capony F, Maudelonde T and et al. Estradiol stimulates cell growth and secretion of precathepsin D and a 120kilodalton protein in the human ovarian cancer cell line BG-1. Journal of Clinical Endocrinology and Metabolism 1992;75(6):1497502

[10]. Langdon SP, Crew AJ, Ritchie AA et al.aGrowth inhibition of oestrogen receptor-positive human ovarian carcinoma by antioestrogensin vitroand in a xenograft model. European Journal of Cancer 1994;30A(5):682-6.

[11]. Slotman BJ \& Rao BR. Ovarian cancer (review): etiology, diagnosis, prognosis, surgery, radiotherapy, chemotherapy and endocrine therapy. Anticancer Res. 1988;8(3):417-34

[12]. Rao BR, Stolman BJ. Ovarian tumors with endocrine manifestations. Curr Ther Endocrinol Metab. 1997;6:260-2.

[13]. Munstedt K, Steen J, Knauf AG, et al. Steroid hormone receptors and long term survival in invasive ovarian cancer. Cancer. 2000 Oct 15;89(8):1783-91.

[14]. Schwartz PE, Gershenson DM, McQuire WP et al. The role of hormonal therapy in the management of ovarian cancer. Controversies in management. New York: Churchill Livingstone, 1998:325-341

[15]. Klinik Jinekolojik Endokrinoloji ve İnfertilite (Leon Speroff ve Marc A.Fritz) 2005; 64-65

[16]. Ahlgren JD, Ellison NM, Gottlieb RJ, et al. Hormonal palliation of chemoresistant ovarian cancer: three consecutive phase II trials of the Mid-Atlantic Oncology Program. J Clin Oncol 1993 Oct;11(10):1957-68.

[17]. Gennatas C, Dardoufas C, Karvouni H, et al. Phase II trial of tamoxifen in patients with advanced epithelial ovarian cancer. Proc Am Soc Clin Oncol 1996; 15:782.

[18]. Hamerlynck JV, Vermorken JB, Van der Burgh ME. Tamoxifen therapy in advanced ovarian cancer. Proc Am Soc Clin Oncol 1985;4:15(abst).

[19]. Schwartz PE, Chambers JT, Kohorn EI, et al. Tamoxifen in combination with cytotoxic chemotherapy in advanced epithelial ovarian cancer. Cancer. 1989;15;63(6):1074-8.

[20]. Markman M, Iseminger KA, Hatch KD, et al. Tamoxifen in platinum-refractory ovarian cancer: a Gynecologic Oncology Group Ancillary Report. Gynecol Oncol. 1996; 62(1):4-6.

[21]. Hatch KD, Beecham JB, Blessing JA, et al. Responsiveness of patients with advanced ovarian carcinoma to tamoxifen. A Gynecologic Oncology Group study of second-line therapy in 105 patients. Cancer. 1991 Jul 15;68(2):269-71.

[22]. Rose FV \& Barnea ER 1996 Response of human ovarian carcinoma cell lines to antiprogestin mifepristone. Oncogene. 1996 Mar 7;12(5):999-1003.

[23]. Benedetti Panici P, Greggi S, Amoroso M, et al. A combination of platinum and tamoxifen in advanced ovarian cancer failing platinum-based chemotherapy: results of Phase II study. Int J Gynecol Cancer. 2001 Nov-Dec;11(6):438-44. 